\title{
263 NECESSITY TO PREPARE AND PROVIDE AN ILLUSTRATED DICTIONARY OF PHYSICAL EDUCATION AND SPORT SCIENCES
}

Vahid Karim Azad Marjani, Seyed Mohammad Marandi, Volga Hovfsepian, Hamidreza Mirsafiyan Faculty of Physical Education and Sport Science, Isfahan University, Isfahan, Iran

\subsection{6/bjsm.2010.078725.263}

The scope of technologies is so miscellaneous in military, security, medical and many other fields. According to the findings, reported by the Chinese institutions for exploration of Taiwanese markets, the ratio of software production volume to the similar amount for hardware in global information industry in 2010 will stand at 2:1; while this ratio has been $6: 4$ in 1997. In producing of this software, all of the available Persian and English language software's were used and the outline of creating Persian-English software was formed, furthermore, according to expansion of sport sciences and specialisation of the domains, it was decided to illustrate the dictionary. As a result the required pictures were provided from available sources and sorted alphabetically. The restoration of information was also done in an alphabetical order from the information bank. The videos and animations were formatted in FLV to occupy less amounts of space when installed. When the user types a word in this software, recalling of sound and image is possible in addition to Persian or English equivalents. More than 1000 images, 600 videos and 3350 words have been installed in the above-mentioned software and need a small space owing to the compressing tools utilised. In joint texts of $\mathrm{PE}$ and sport sciences there are some terms from all of the branches that mislead the specialists in translation of them. The present software is so comprehensive that solves such problems. Survey of this software demonstrates its advantages such as simplicity of use and availability of equivalents. 